\title{
ARTICLE
}

Epidemiology

\section{Benefits of switching from guaiac-based faecal occult blood to faecal immunochemical testing: experience from the Wallonia-Brussels colorectal cancer screening programme}

\author{
Feng Guo $\mathbb{D D}^{1,2}$, Isabel De Brabander ${ }^{3}$, Julie Francart ${ }^{3}$, Michel Candeur ${ }^{4}$, Marc Polus ${ }^{5}$, Liesbet Van Eycken ${ }^{3}$ and Hermann Brenner ${ }^{1,6,7}$
}

BACKGROUND: Faecal immunochemical tests (FITs) have replaced guaiac-based faecal occult blood test (gFOBTs) in several colorectal cancer (CRC) screening programmes. We aimed to evaluate the benefits of this transition based on the Wallonia-Brusselsorganised CRC screening programme.

METHODS: A total of 1,569,868 individuals aged 50-74 years, who were invited to screening during 2009-2017, were studied by linking their screening records with insurance, pathology and cancer data in the Belgian Cancer Registry. We compared neoplasm detection rates and positive predictive values (PPVs) of gFOBT and FIT at $15 \mu \mathrm{g}$ haemoglobin per gram cut-off in screen-naive individuals. We furthermore examined the incidence rates of interval cancer in GFOBT- and FIT-based screening programme. RESULTS: Advanced neoplasms were detected less frequently by gFOBT $(0.8 \%)$ than by FIT $(1.3 \%)$, with a difference of $0.5 \%(P<$ 0.01). PPVs were lower for gFOBT (15.1\%) than for FIT (21.7\%) for advanced neoplasms (difference $6.6 \%, P<0.01$ ). Compared to participants with negative gFOBT, those with negative FIT were $77 \%$ less likely to develop interval cancer (incidence rate ratio 0.23 , 95\% confidence interval 0.16-0.33).

CONCLUSION: Our study demonstrated that in an organised CRC screening programme, replacing gFOBT with FIT improved neoplasm detection rate and substantially reduced interval cancer incidence.

British Journal of Cancer (2020) 122:1109-1117; https://doi.org/10.1038/s41416-020-0754-5

\section{BACKGROUND}

Colorectal cancer (CRC) is the third most commonly diagnosed cancer and the second leading cause of cancer-related mortality worldwide. ${ }^{1}$ A substantial proportion of CRC deaths can be prevented by screening. Over many years, the most commonly used non-invasive test for CRC screening has been the guaiacbased faecal occult blood test (gFOBT). Offering annual gFOBT screening has been shown to reduce CRC mortality by up to $30 \%$ in randomised clinical trial, although the sensitivity of this test to detect CRC and its precursors has been very limited. ${ }^{2,3}$

In recent years, faecal immunochemical tests (FITs), which were shown to be more sensitive for colorectal adenomas and CRCs, ${ }^{4-9}$ have replaced gFOBT or have been newly introduced in many CRC screening programmes. ${ }^{8,10}$ However, according to a recent European Union-funded report on cancer screening programmes, in some countries, such as Croatia, Finland, Latvia and Sweden, gFOBTs were still offered as primary CRC screening tests. ${ }^{11}$ Previous studies have demonstrated that replacing gFOBT by FIT in pilot screening programmes markedly increased the participation rate and yield of advanced neoplasia. ${ }^{12-14}$ Although implementation of FIT-based screening has been widely recommended, empirical evidence on the impact of switching from
gFOBT- to FIT-based screening on key outcome variables of screening on the population level is yet very limited.

In the Walloon and Brussels-capital region of Belgium, an organised gFOBT-based CRC screening programme had been fully implemented since March 2009 and transited to FIT-based screening in March 2016. In this study, we aimed to evaluate the neoplasm detection rates, positive predictive values (PPVs), characteristics of screen- and non-screen-detected cancers and incidence rates of interval cancer in the Wallonia-Brussels-organised CRC screening programme before and after this transition.

\section{METHODS}

Wallonia-Brussels CRC screening programme

Since March 2009, an organised gFOBT-based screening programme has been fully implemented in Wallonia and Brussels. The screening programme covers the population from the French- and German-speaking community of Belgium. Women and men aged 50-74 years were invited biennially and asked to collect a test kit for the purpose of CRC screening from their general practitioners (GPs), together with a prepaid envelope for returning the completed test kit. In March 2016, the Wallonia-Brussels CRC

\footnotetext{
${ }^{1}$ Division of Clinical Epidemiology and Aging Research, German Cancer Research Center (DKFZ), Heidelberg, Germany; ${ }^{2}$ Medical Faculty Heidelberg, University of Heidelberg, Heidelberg, Germany; ${ }^{3}$ Belgian Cancer Registry (BCR), Brussels, Belgium; ${ }^{4}$ Community Reference Center for Cancer Screening (Wallonia), Mont-Saint-Guibert, Belgium; ${ }^{5}$ Department of Gastroenterology, University Hospital of Liège, Liège, Belgium; ${ }^{6}$ Division of Preventive Oncology, German Cancer Research Center (DKFZ) and National Center for Tumor Diseases (NCT), Heidelberg, Germany and ${ }^{7}$ German Cancer Consortium (DKTK), German Cancer Research Center (DKFZ), Heidelberg, Germany Correspondence: Hermann Brenner (h.brenner@dkfz-heidelberg.de)
}

Received: 26 July 2019 Revised: 20 January 2020 Accepted: 30 January 2020

Published online: 18 February 2020 
1110

screening guideline was officially updated by replacing gFOBT with FIT. For both the gFOBT- and FIT-based screening programme, no specific reminders were sent to persons who had not collected a test kit from their GPs or had not returned a completed test after receiving a test kit.

The gFOBT used in Wallonia and Brussels was Hemoccult II (Beckman Coulter). For FIT, an automated quantitative FIT (OCSensor, Eiken Chemical, Tokyo, Japan) was offered. The analyses of returned gFOBT and FIT samples were done at the Walloon Central Laboratory (Mont-Saint-Guibert, Belgium). Positivity of gFOBT was defined as blue discoloration of any of the six returned stool samples (on three cards) within 30-60 s after applying the developing solution. The positivity threshold for FIT was set at $15 \mu \mathrm{g}$ haemoglobin per gram $(\mathrm{Hb} / \mathrm{g})$ faeces. Detailed information on sample collection and handling, laboratory analysis, quality management and data handling according to the checklist for FITs for $\mathrm{Hb}$ evaluation reporting (FITTER) ${ }^{15}$ are provided in the Supplementary Appendix.

The GPs of the screening participants were informed on the test result within 5 days after analysis. Participants with negative result were suggested to attend screening again after 2 years. A major change regarding subsequent screening invitation was made in March 2015. Before then, participants with prior negative result were invited again by a letter without inclusion of a faecal test kit, whereas an invitation letter along with a test kit was sent to them from March 2015 on. Participants with a positive result were advised to undergo colonoscopy. Colonoscopies for follow-up of positive gFOBT/FIT results were conducted by gastroenterologists, specialists in internal medicine, or surgeons in hospitals or private practices. $^{16}$

The faecal test (either gFOBT or FIT) was offered free of charge for all individuals invited for screening, while the advised followup colonoscopy, medical care and treatment were partially reimbursed by the health insurance company. The proportion of reimbursement varied according to personal circumstances (health status, employment status, financial status, etc.).

Databases for this study

The data used in this study included individuals aged 50-74 years who were invited for CRC screening between March 2009 and June 2017. Overall, $1,569,868$ individuals $(195,170$ persons screened at least once, and 1,374,698 persons never screened) were included in our analysis. Information on screening invitation, participation, type of faecal test and test results were obtained from the Walloon screening organisation. Although FIT has been officially offered instead of gFOBT since March 2016 in Wallonia and Brussels, due to inventory management many clinical settings continued offering surplus gFOBT kits until June 2017. Additionally, a few GPs have provided FIT to attendees since January 2014 on the background of a pilot study, which aimed to adapt to the changes in switching screening tests. The present study therefore includes gFOBTs and FITs, which have been offered between March 2009 and June 2017, and between January 2014 and June 2017, respectively.

Data on adherence to, date and completeness of follow-up colonoscopy were obtained from the Intermutualistic Agency (IMA) database, which covers nearly $99 \%$ of the Belgian population and includes data collected in the framework of the compulsory Belgian health care and benefits insurance programme, on reimbursed medication and the use of reimbursed health services (up to date until December 2017 by the time of this analysis).

After obtaining the information on use of screening test and follow-up examination, the study population was linked to the Central Cyto-Histopathology (CHP) and Cancer Registry (CR) databases in the Belgian CR (BCR). The BCR is engaged in the assessment of quality and effectiveness of the Belgian national organised screening programmes for colorectal, cervical and breast cancer. The CHP database documents the histopathological results of colorectal samples from individuals with or without screening and covers results from all pathological laboratories (pathology network) in Belgium. All data that enters the CHP database are submitted to an extended set of automated and manual validation procedures to ensure validity and quality of the data. The histopathologic information was used to validate CRC diagnosis. Approximately $99 \%$ of CRC cases registered at the BCR have been microscopically verified. ${ }^{17}$ Data on characteristics of tumour (location and stage) and patient (age and sex) for all identified CRC cases between March 2009 and December 2016 were collected from the CR database, and these characteristics (except for tumour stage) for CRCs diagnosed in 2017 were ascertained from the CHP database.

Morphology and topography of colorectal lesions were coded with the International Classification of Diseases for Oncology, 3rd version. Neoplasms were defined as proximal if located from the caecum to the splenic flexure and as distal otherwise. Tumour stage was classified according to the TNM (tumour node metastasis) 6th (year of diagnosis: 2009) or 7th (year of diagnosis: 2010-2017) edition. In our study, advanced adenomas were defined as adenomas with tubulovillous or villous histology, or those with high-grade dysplasia. Patients with intramucosal carcinoma or carcinoma in situ were classified as having highgrade dysplasia. Cancers were defined by the invasion of malignant cells beyond the muscularis mucosa. Each patient was classified based on the most advanced lesion. An overview on the time periods covered by the various databases and used in our analysis is given in Supplementary Fig. 1 .

Screen- and non-screen-detected CRC

Screen-detected CRCs were defined as cancers diagnosed within 6 months of colonoscopy following a positive gFOBT or FIT result. Non-screen-detected CRCs were defined as CRCs that were not diagnosed within the screening programme and divided into four groups: (1) gFOBT/FIT interval cancers, defined as cancers diagnosed between screening rounds after negative gFOBT/FIT and before the next recommended test. (2) Colonoscopy interval cancers, defined as cases in which a follow-up colonoscopy was performed more than 6 months before CRC diagnosis. (3) CRCs in colonoscopy non-compliers, defined as cancers diagnosed in those who received a positive gFOBT/FIT result, but did not undergo a follow-up colonoscopy. (4) CRCs in non-attendees, defined as cancers diagnosed in those who did not collect a test kit from their GP or did not return the completed gFOBT/FIT kit. The rationale of using 6 months as a time window to distinguish screen-detected cancer and colonoscopy interval cancer is based on the assumption that CRCs suspected/detected at colonoscopy would be diagnosed within 6 months of the index procedure, and this definition has been previously used in several studies. ${ }^{18-20}$

\section{Statistical analysis}

In the first set of analyses, we included screen-naive individuals who only attended one screening round to compare test positivity, colonoscopy adherence, detection rate and PPV of gFOBT with FIT. The comparison of participation rates in gFOBT and FIT screening was not included in the primary analysis, as the invitation letter did not specifically address the type of faecal test. We calculated the positivity rate as the number of persons with a positive gFOBT/FIT relative to the number of persons returning a gFOBT/FIT. The rate of colonoscopy adherence was calculated as the number of participants undergoing follow-up colonoscopy relative to the number of positive tests. The detection rate was calculated as the number of participants with lesions relative to the number of participants screened. The PPV was calculated as the number of detected lesions relative to the total number of follow-up colonoscopies. Furthermore, sex- and age-stratified detection rates for any advanced neoplasm (advanced adenoma 
or $\mathrm{CRC}$ ) among gFOBT and FIT users were calculated. Rates and rate differences of above-mentioned outcomes were calculated, and all percentages were reported with $95 \%$ confidence interval $(\mathrm{Cl})$.

In the second set of analyses, we evaluated the characteristics of screen- and non-screen-detected CRCs as well as the incidence rates of interval cancer in gFOBT- and FIT-based screening programmes. The proportion of gFOBT/FIT interval cancers was calculated by dividing the number of $\mathrm{gFOBT} / \mathrm{FIT}$ interval cancers by the sum of screen-detected and gFOBT/FIT interval cancers. Descriptive analysis was performed on CRC patient (sex, age) and tumour (location, stage) characteristics for gFOBT and FIT users separately. Differences in proportions between CRC groups were assessed using the $X^{2}$ test. Incidence rates of gFOBT and FIT interval cancer were calculated per 10,000 person-years. The follow-up time period was calculated as the time between negative test and interval cancer occurrence or censoring (either by December 2017 or by 2-year follow-up), whichever came first. An incidence rate ratio (IRR) and the corresponding $95 \% \mathrm{Cl}$ were calculated to compare the incidence rates of gFOBT and FIT interval cancers.

To fully reflect the number of advanced adenomas and CRCs that have been detected in the gFOBT- or FIT-based screening programme, compliance to follow-up colonoscopy was considered as colonoscopy conducted within 24 months after positive gFOBT/FIT. We additionally reported results on colonoscopy completion rate, detection rate, PPV, characteristics of screendetected CRC and patients by considering follow-up colonoscopy as colonoscopy conducted within 6 months after positive faecal test.

All analyses were performed with SAS Enterprise Guide version 7.1 (SAS Institute, Cary, NC, USA). Two-sided $P$ values $<0.05$ were considered statistically significant.

\section{RESULTS}

Test positivity, detection rate and PPV

From 195,170 screening participants, 122,507 individuals attended only one screening round, among whom 94,290 used gFOBT and 28,217 used FIT (Fig. 1). The mean age was similar for gFOBT users (mean: $60.9 \pm$ standard deviation: 7.3 years) and FIT users (59.9 \pm 6.9 years). Of the gFOBT and FIT participants, $5645(6.0 \%)$ and $2185(7.7 \%)$ tested positive, resulting in a difference in positivity rate of $1.7 \%(95 \% \mathrm{Cl}$ : $1.4-2.1$; Table 1$)$. Follow-up colonoscopy completion rates were higher for gFOBT (84.6\%) vs. FIT (78.5\%), with a difference of $-6.1 \%(95 \% \mathrm{Cl}:-8.0$ to -4.0$)$. Advanced neoplasms were found in 720 of the gFOBT participants $(0.8 \%)$ and in 373 of the FIT participants (1.3\%), with a difference of $0.5 \%$ (95\% Cl: 0.4-0.7). PPVs were also lower for gFOBT than for FIT: $6.5 \%$ vs. $6.9 \%$ for CRC (difference $0.4 \%, 95 \% \mathrm{Cl}:-1.0$ to 1.8 ), and $15.1 \%$ vs. $21.7 \%$ for any advanced neoplasm (difference $6.6 \%, 95 \%$ Cl: 4.5-8.9). The PPVs for above-mentioned outcomes remained almost unchanged when considering follow-up colonoscopy as colonoscopy conducted within 6 months after positive faecal test (Supplementary Table 1).

The detection rates were higher for men than for women in both gFOBT- and FIT-based screening programmes, but the detection rates were consistently higher in the latter screening programme (Table 2). No clear trend in detection rates with age was seen for gFOBT participants. In contrast, detection rates among participants screened with FIT rose across age categories, increasing from $0.8 \%$ at age $50-59$ years to $2.3 \%$ at age $\geq 70$ years for any advanced neoplasm.

Proportion of screen- and non-screen-detected CRCs During March 2009-June 2017, a total of 1,569,868 individuals were eligible for CRC screening, of whom 195,170 (12.4\%) participated at least once (Fig. 1). Of these, 14,196 (7.3\%) had a positive faecal test and 11,878 subsequently underwent colonoscopy $(83.7 \%$ compliance). In the total population of $1,569,868$, CRC was detected in 1213 screening participants and in 13,973 who never participated. The CRCs detected in the 1213 participants included 590 screen-detected CRCs (48.6\%), 535 gFOBT/FIT interval cancers $(44.1 \%), 31$ colonoscopy interval cancers (2.6\%) and $57 \mathrm{CRCs}$ in colonoscopy non-compliers (4.7\%). While the proportion of gFOBT interval cancer was $54.1 \%$ (503 interval cancers vs. 426 screen-detected CRCs), reflecting a gFOBT sensitivity for detecting CRC of $\sim 46 \%$, the proportion of FIT interval cancer was $16.3 \%$ (32 interval cancers vs. 164 screendetected CRCs), reflecting a FIT sensitivity for detecting CRC of $\sim 84 \%$. When considering follow-up colonoscopy as colonoscopy conducted within 6 months after positive faecal test, the proportions of interval cancer were essentially unchanged (55.2\% for gFOBT and $16.8 \%$ for FIT, Supplementary Table 2 )

Characteristics of CRC patients

Table 3 shows the characteristics of CRC patients within the screening programme. Screen-detected cancer, gFOBT/FIT interval

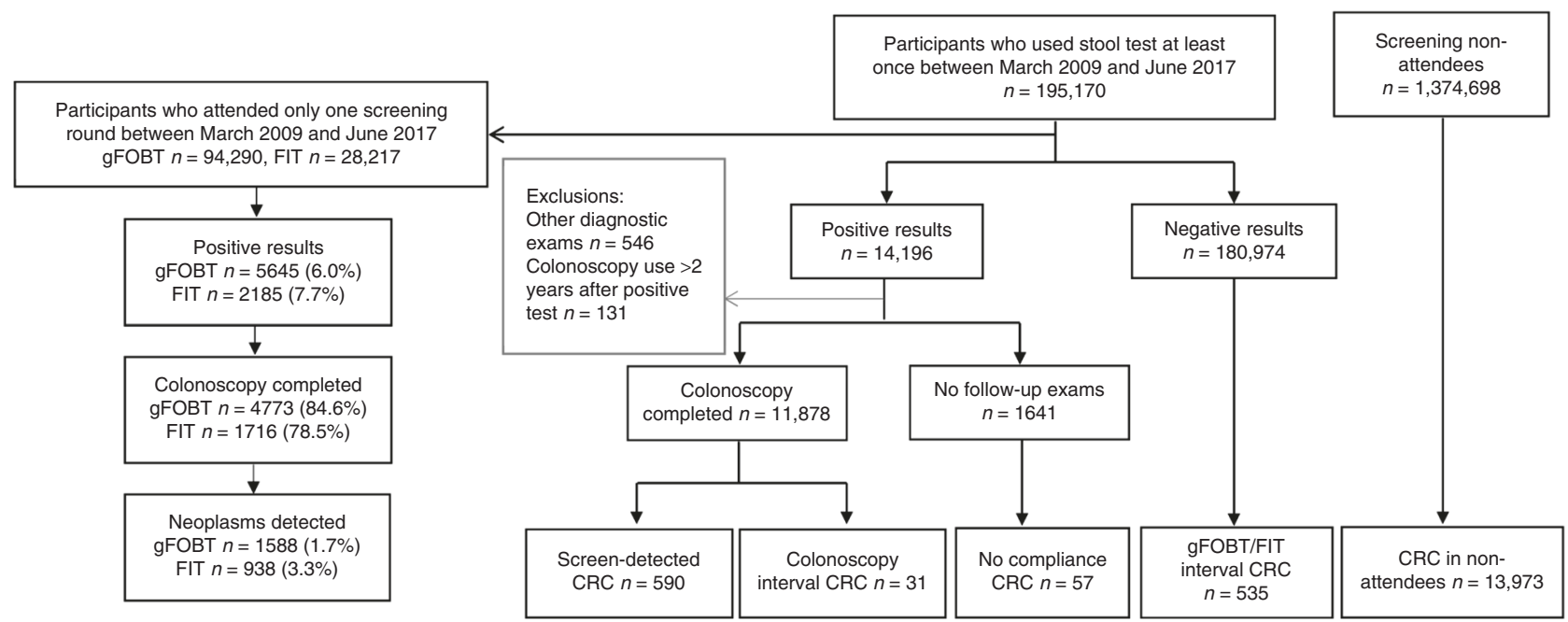

Fig. 1 Flow diagram for analyses on detection rate, screen- and non-screen-detected CRCs in the Wallonia-Brussels-organised gFOBTand FIT-based screening programme. 
Table 1. Indicators of test performance of gFOBT vs. FIT.

\begin{tabular}{|c|c|c|c|c|c|}
\hline & \multicolumn{4}{|c|}{ Type of stool test used for screening } & \multirow{2}{*}{$\begin{array}{l}\text { Difference }^{a} \\
\%(95 \% \mathrm{Cl})\end{array}$} \\
\hline & $n$ & $\%(95 \% \mathrm{Cl})$ & $n$ & $\%(95 \% \mathrm{Cl})$ & \\
\hline Positive gFOBT/FIT results & 5645 & $6.0(5.8-6.1)$ & 2185 & $7.7(7.4-8.1)$ & $1.7(1.4-2.1)$ \\
\hline Colonoscopy completion & 4773 & $84.6(83.6-85.5)$ & 1716 & $78.5(76.8-80.3)$ & $-6.1(-8.0$ to -4.1$)$ \\
\hline \multicolumn{6}{|l|}{ Detection rate } \\
\hline Advanced adenoma & 410 & $0.4(0.4-0.5)$ & 254 & $0.9(0.8-1.0)$ & $0.5(0.3-0.6)$ \\
\hline Any advanced neoplasm & 720 & $0.8(0.7-0.8)$ & 373 & $1.3(1.2-1.5)$ & $0.5(0.4-0.7)$ \\
\hline Non-advanced adenoma & 868 & $0.9(0.9-1.0)$ & 565 & $2.0(1.8-2.2)$ & $1.1(0.9-1.3)$ \\
\hline Any adenoma & 1278 & $1.4(1.3-1.4)$ & 819 & $2.9(2.7-3.1)$ & $1.5(1.3-1.8)$ \\
\hline \multicolumn{6}{|l|}{ Positive predictive value ${ }^{b}$} \\
\hline
\end{tabular}

Table 2. Detection rates of gFOBT and FIT for any advanced neoplasm, by sex and age.

\begin{tabular}{|c|c|c|c|c|c|}
\hline & \multicolumn{4}{|c|}{ Type of stool test used for screening } & \multirow{3}{*}{$\begin{array}{l}\text { Difference }^{a} \\
\%(95 \% \mathrm{Cl})\end{array}$} \\
\hline & \multicolumn{2}{|c|}{$\begin{array}{l}\text { gFOBT Total } \\
N=94,290\end{array}$} & \multicolumn{2}{|c|}{$\begin{array}{l}\text { FIT Total } \\
N=28,217\end{array}$} & \\
\hline & $n$ & $\%(95 \% \mathrm{Cl})$ & $n$ & $\%(95 \% \mathrm{Cl})$ & \\
\hline Total & 720 & $0.8(0.7-0.8)$ & 373 & $1.3(1.2-1.5)$ & $0.5(0.4-0.7)$ \\
\hline \multicolumn{6}{|l|}{ Sex } \\
\hline Male & 476 & $1.1(1.0-1.2)$ & 243 & $1.8(1.6-2.0)$ & $0.7(0.4-0.9)$ \\
\hline Female & 244 & $0.5(0.4-0.5)$ & 130 & $0.9(0.7-1.1)$ & $0.4(0.3-0.6)$ \\
\hline \multicolumn{6}{|l|}{ Age } \\
\hline $50-59$ years & 235 & $0.5(0.5-0.6)$ & 127 & $0.8(0.7-1.0)$ & $0.3(0.1-0.4)$ \\
\hline $60-69$ years & 355 & $1.0(0.9-1.1)$ & 184 & $1.8(1.6-2.1)$ & $0.8(0.5-1.1)$ \\
\hline$\geq 70$ years & 130 & $0.8(0.6-0.9)$ & 62 & $2.3(1.7-2.8)$ & $1.5(1.0-2.1)$ \\
\hline \multicolumn{6}{|c|}{$\begin{array}{l}\mathrm{Cl} \text { confidence interval, } F I T \text { faecal immunochemical test, } g F O B T \text { guaiac-based } \\
\text { faecal occult blood test. } \\
\text { a'Differences with a } 95 \% \mathrm{Cl} \text { completely lower or higher than } 0 \text { are } \\
\text { statistically significant, which means that the } P \text { value does not exceed } 0.05 \text {. }\end{array}$} \\
\hline
\end{tabular}

cancer, colonoscopy interval cancer and CRC in non-attendees were found predominantly in men and among people aged 60-69 years in the overall screening programme; however, CRC cases among those who did not adhere to follow-up colonoscopy were quite equally distributed between sexes and across age groups. While significant differences were observed between patients with screen- and non-screen-detected CRCs for age $(P=0.013)$ and sex $(P=0.014)$ in the overall screening programme, there were only significant sex differences between the two groups of patients in gFOBT-based screening $(P=0.002)$ and only significant age differences in FIT-based screening $(P=0.003)$.

Tumour location and stage distribution

Tumour location and CRC stage distribution are described in Table 4. In the gFOBT-based screening programme, colonoscopy interval cancers $(40.7 \%)$ were more commonly located in the proximal colon than screen-detected CRCs (28.7\%), gFOBT interval cancers (32.6\%) and CRCs among colonoscopy non-compliers (14.3\%). While screen-detected CRCs (30.8\%) and FIT interval cancers $(26.9 \%)$ were less commonly found in the proximal colon, most colonoscopy interval cancers $(66.7 \%)$ and CRCs in colonoscopy non-compliers $(66.7 \%)$ were located proximally in the FIT screening subgroups. The stage distribution differed significantly among screen- and non-screen-detected groups, with more favourable stages in participants with screen-detected CRC $(P<$ 0.001). Stage distribution was similar for gFOBT/FIT interval cancers and CRCs in non-attendees $(P=0.624)$. While a large proportion of early-stage screen-detected CRCs was observed in both gFOBT- and FIT-based screening programmes, the proportion of stage I cancers was much lower among CRCs detected by gFOBT than by FIT (39.3\% vs. $49.3 \%)$.

Incidence rate of gFOBT/FIT interval cancer

Among $535 \mathrm{gFOBT} / \mathrm{FIT}$ interval cancers, 503 cases occurred during 236,102 person-years after a negative gFOBT and 32 cases occurred during 64,750 person-years after a negative FIT (Table 5). The incidence rates of gFOBT and FIT interval cancers were 21.3 (95\% Cl: 19.4-23.2) and 4.94 (95\% Cl: 3.23-6.65) per 10,000 personyears, respectively. Compared to participants with a negative gFOBT, those with a negative FIT were $77 \%$ less likely to develop an interval cancer (IRR $0.23,95 \% \mathrm{Cl}$ : 0.16-0.33).

\section{DISCUSSION}

In this population-based study, we compared important outcome variables of gFOBT- and FIT-based screening in the Wallonia-Brussels-organised CRC screening programme with comprehensive access to the dynamic data in regard to screening, insurance claims, pathology and cancer registration. This study showed that the detection rate and PPV for advanced neoplasms were approximately one third lower in gFOBT users compared to FIT users. Furthermore, the proportion and incidence rate of interval cancers were approximately four times higher with gFOBT than with FIT.

A major advantage of FIT is that it provides a quantitative result, which allows shifting the cut-off value of the test and adapting it to the specific needs of the population to be screened. ${ }^{21-23} \mathrm{~A}$ recent meta-analysis demonstrated that FIT sensitivity for detection of CRC increased from $69 \%$ at cut-offs $10-20 \mu \mathrm{g} \mathrm{Hb} / \mathrm{g}$ to $80 \%$ 
Table 3. Characteristics of CRC patients in the Wallonia-Brussels-organised gFOBT- and FIT-based screening programme.

\begin{tabular}{|c|c|c|c|c|c|c|c|}
\hline & \multirow[t]{2}{*}{ Screen-detected CRC } & \multicolumn{5}{|c|}{ Non-screen-detected CRC } & \multirow[t]{2}{*}{$P$ value $^{\mathrm{b}}$} \\
\hline & & gFOBT/FIT & Colonoscopy & $\begin{array}{l}\text { No colonoscopy } \\
\text { compliance }\end{array}$ & CRC in non-attendees ${ }^{a}$ & Overall & \\
\hline \multicolumn{8}{|l|}{ gFOBT + FIT } \\
\hline Total CRC & 590 & 535 & 31 & 57 & 13973 & 14,596 & \\
\hline \multicolumn{8}{|l|}{ Age, $n$ (\%) } \\
\hline $60-69$ years & $303(51.4)$ & $270(50.5)$ & $15(48.4)$ & $19(33.3)$ & $6291(45.0)$ & $6595(45.2)$ & \\
\hline$\geq 70$ years & $150(25.4)$ & $156(29.1)$ & $12(38.7)$ & $20(35.1)$ & $4010(28.7)$ & $4198(28.8)$ & \\
\hline \multicolumn{8}{|l|}{ Sex, $n(\%)$} \\
\hline Male & $370(62.7)$ & $287(53.6)$ & $22(71.0)$ & $28(49.1)$ & $8072(57.8)$ & 8409 (57.6) & 0.014 \\
\hline Female & $220(37.3)$ & $248(46.4)$ & $9(29.0)$ & $29(50.9)$ & $5901(42.2)$ & $6187(42.4)$ & \\
\hline \multicolumn{8}{|l|}{ gFOBT } \\
\hline $60-69$ years & $215(50.5)$ & $260(51.7)$ & $15(53.6)$ & $19(37.3)$ & - & $294(50.5)$ & \\
\hline$\geq 70$ years & $102(23.9)$ & $139(27.6)$ & $10(35.7)$ & $18(35.3)$ & - & $167(28.7)$ & \\
\hline \multicolumn{8}{|l|}{ Sex, $n(\%)$} \\
\hline Male & $273(64.1)$ & $270(53.7)$ & $21(75.0)$ & $24(47.1)$ & - & $315(54.1)$ & 0.002 \\
\hline Female & $153(35.9)$ & $233(46.3)$ & $7(25.0)$ & $27(52.9)$ & - & $267(45.9)$ & \\
\hline \multicolumn{8}{|l|}{ FIT } \\
\hline Total CRC & 164 & 32 & 3 & 6 & - & 41 & \\
\hline \multicolumn{8}{|l|}{ Age, $n(\%)$} \\
\hline 50-59 years & $28(17.0)$ & 5 (15.6) & $1(33.3)$ & $4(66.7)$ & - & $10(24.4)$ & 0.003 \\
\hline $60-69$ years & $88(53.7)$ & $10(31.3)$ & $0(0)$ & $0(0)$ & - & $10(24.4)$ & \\
\hline
\end{tabular}

at cut-offs $\leq 10 \mu \mathrm{g} \mathrm{Hb} / \mathrm{g}^{24}$ Consistently, sensitivity for detection of advanced adenomas increased from 21 to $31 \%$ at these cut-off values. While lower cut-off levels for referral to colonoscopy may be more effective when colonoscopy capacity is sufficient in screening programmes, higher cut-offs might be appropriate for programmes with limited colonoscopy resources. Furthermore, in contrast to gFOBT, FIT detects human $\mathrm{Hb}$ free of cross-reactivity with animal $\mathrm{Hb}$ and other dietary constituents. Additional advantages of FIT include the possibility of high-throughput automated processing as well as user-friendly application and higher adherence. $7,13,14,25,26$ Our results demonstrate that, at comparable positivity rates $(6.0 \%$ for gFOBT vs. $7.7 \%$ for FIT), gFOBT is much less sensitive than FIT for clinically relevant colorectal neoplasms, which suggests that programmes switching from gFOBT to FIT will achieve better outcomes, even if using a FIT positivity threshold (at $15 \mu \mathrm{g} \mathrm{Hb} / \mathrm{g}$ cut-off) that yields similar positivity rates as the gFOBT. Furthermore, given that the positivity rate of FIT among individuals previously screened with gFOBT (8.0\%) was nearly the same as among FIT users without previous screening (7.7\%), overall demands on colonoscopy capacity may not dramatically increase when switching to a FITbased CRC screening programme.
In this study, there was no difference between gFOBTs and FITs concerning the higher detection rates among male participants. However, in contrast to gFOBT, the detection rates of advanced neoplasms strongly increased with age among FIT participants, suggesting that advantages of FIT-based screening over gFOBTbased screening are particularly large in the older age groups, in which prevalence and incidence of CRC are higher than in younger age groups.

Due to the lower sensitivity of gFOBT than FIT in detecting advanced neoplasia, interval cancer rates are expected to be lower in the latter screening programme. Studies from several European countries, including Spain, Denmark, France, Sweden and Scotland, have reported high proportions of interval cancer in the programmes offering gFOBT as an initial screening test (range: 48-58\%). ${ }^{27-31}$ Reported proportions were much lower in studies from the Netherlands (23\%), ${ }^{32}$ Slovenia (14\%), ${ }^{33}$ and Italy (10\%), where FIT is used as primary CRC screening test and positivity thresholds are set at levels $10-20 \mu \mathrm{g} \mathrm{Hb} / \mathrm{g}$ faeces. Notably, with a high cut-off of $80 \mu \mathrm{g} \mathrm{Hb} / \mathrm{g}$ faeces, the proportion of FIT interval cancer was remarkably similar to that seen with gFOBT. ${ }^{35}$ In this study, we demonstrated that the proportion of interval cancers dropped from $54.1 \%$ in gFOBT-based screening to $16.3 \%$ in 
Benefits of switching from guaiac-based faecal occult blood to faecal...

$\mathrm{F}$ Guo et al.

Table 4. Tumour location and stage distribution of CRCs in the Wallonia-Brussels-organised gFOBT- and FIT-based screening programme.

Screen-detected CRC Non-screen-detected CRC

\begin{tabular}{ll}
\hline Interval CRC & No colonoscopy compliance CRC in non-attendees Overall \\
gFOBT/FIT Colonoscopy &
\end{tabular}

gFOBT + FIT

Total CRC

590

535

31

57

13,973

14,596

Tumour site, $n(\%)$

Proximal $\quad 159(29.2)$

$162(32.3) \quad 13(43.3) \quad 11(20.0)$

$3672(28.6)$

3858 (28.7)

0.788

Distal $385(70.8)$

339 (67.7) $17(56.7)$

$44(80.0)$

$9187(71.4)$

$9587(71.3)$

Stage, $n(\%)$

I $180(40.9)$

II $122(27.7)$

$98(23.8) \quad 5(25.0)$

9 (19.1)

$2164(21.2)$

$2276(21.3)<0.001$

III $100(22.7)$

$106(25.8) \quad 5(25.0)$

9 (19.1)

$2804(27.5)$

$2924(27.4)$

$114(27.7) \quad 7(35.0) \quad 21(44.7)$

2889 (28.3)

3031 (28.4)

$93(22.6) \quad 3(15.0) \quad 8(17.0)$

$2339(22.9)$

$2443(22.9)$

gFOBT $^{\text {b }}$

Total CRC $\quad 426$

$503 \quad 28 \quad 5$

51

582

Tumour site, $n$ (\%)

Proximal $\quad 119(28.7)$

$155(32.6) \quad 11(40.7) \quad 7(14.3)$

Distal $295(71.3)$

$320(67.4) \quad 16(59.3) \quad 42(85.7)$

Stage, $n(\%)$

I $145(39.3)$

$96(23.7) \quad 5(25.0) \quad 9(19.6)$

$106(26.2) \quad 5(25.0) \quad 8(17.4)$

$102(27.6)$

$114(28.1) \quad 7(35.0) \quad 21(45.7)$

$\begin{array}{ll} & 85(23.0) \\ \text { IV } & 37(10.0)\end{array}$

$89(22.0) \quad 3(15.0)$

8 (17.4)

FIT $^{\mathrm{c}}$

Total CRC 164

$32 \quad 3$

6

Tumour site, $n(\%)$

$\begin{array}{ll}\text { Proximal } & 40(30.8) \\ \text { Distal } & 90(69.2)\end{array}$

7 (26.9) $\quad 2(66.7)$

$4(66.7)$

$19(73.1) \quad 1(33.3) \quad 2(33.3)$

Stage, $n(\%)$

I $35(49.3)$

II $20(28.2)$

III $15(21.1)$

IV 1 (1.4)

$2(33.3) \quad 0(0) \quad 0(0)$

$0(0) \quad 0(0) \quad 1(100)$

$0(0) \quad 0(0) \quad 0(0)$

$4(66.7) \quad 0(0) \quad 0(0)$

$173(31.4)$

$378(68.6)$

$110(23.4)$

$119(25.3)$

$142(30.1)$

$100(21.2)$

41

$13(37.1)$

$22(62.9)$

$2(28.6)$

$<0.001$

$C R C$ colorectal cancer, FIT faecal immunochemical test, gFOBT guaiac-based faecal occult blood test.

${ }^{a} P$ value refers to the difference between screen-detected and overall non-screen-detected CRCs.

bunspecific location for screen-detected CRC, gFOBT interval cancer, colonoscopy interval cancer and CRCs in colonoscopy non-compliers are $n=12,28,1$ and 2, respectively. Unspecific/missing stage for screen-detected CRC, gFOBT interval cancer, colonoscopy interval cancer and CRCs in colonoscopy non-compliers are $n=57,98,8$ and 5 , respectively.

'Unspecific location for screen-detected CRC and FIT interval cancer are $n=34$ and 6, respectively. Unspecific/missing stage for screen-detected CRC, FIT interval cancer, colonoscopy interval cancer and CRCs in colonoscopy non-compliers are $n=93,26,3$ and 5, respectively.

Table 5. Incidence rate of gFOBT and FIT interval cancer in the Wallonia-Brussels-organised screening programme.

\begin{tabular}{llllll}
\hline Screening test & $\begin{array}{l}\text { Interval } \\
\text { cancer cases }\end{array}$ & $\begin{array}{l}\text { Participants with negative } \\
\text { gFOBT/FIT }\end{array}$ & $\begin{array}{l}\text { Person- } \\
\text { years }\end{array}$ & $\begin{array}{l}\text { Incidence rate per 10,000 person- } \\
\text { years (95\% Cl) }\end{array}$ & $\begin{array}{l}\text { Incidence rate ratio (95\% Cl) } \\
\text { gFOBT }\end{array}$ \\
\hline FIT & 32 & 120,008 & 236,102 & $21.3(19.4-23.2)$ & Reference \\
\hline
\end{tabular}

Cl confidence interval, FIT Faecal immunochemical test, gFOBT guaiac-based faecal occult blood test.

FIT-based screening at the cut-off of $15 \mu \mathrm{g} \mathrm{Hb} / \mathrm{g}$ faeces, with incidence rates of interval cancers falling from 21 to 5 cases per 10,000 person-years.

In the evaluation of gFOBT/FIT interval cancer rates, a minimum 2-year follow-up should be set up to observe all the potential interval cancers after a negative screening test. ${ }^{36}$ Although our study is based on a large number of person-years, we acknowledge the possibility of underestimation of gFOBT/FIT interval cancers due to insufficient follow-up time for those screened in the latter 2016 and in 2017. However, the proportion of FIT 
interval cancers observed in our study (16\%) was very close to the proportion observed within the Flemish FIT-based screening programme (18\%), ${ }^{37}$ which is another fully established organised screening programme in Belgium where FIT has been offered since October 2013, suggesting the potential underestimation to be small.

In the Wallonia-Brussels screening programme, most screendetected CRCs, gFOBT/FIT interval cancers, CRCs in colonoscopy non-compliers and CRCs among non-attendees were located in the distal colon or rectum, whereas colonoscopy interval cancers tended to be located in the proximal colon. In our study, 31 colonoscopy interval cancers were detected and the majority (68\%) occurred within 3 years after negative colonoscopy. Since colonoscopy interval cancers were more commonly found in the proximal colon, some procedural issues are needed to consider, such as inadequate bowel preparation and incompleteness of colonoscopy. ${ }^{38-40}$ While gFOBT/FIT interval cancer rates are related to test sensitivity, colonoscopy interval cancer may partly reflect colonoscopy quality. Both cancers should be carefully monitored when implementing a CRC screening programme.

In addition, 57 participants who refused to undergo colonoscopy after a positive gFOBT/FIT were later diagnosed with CRC. Unfortunately, no information on the reason for failing to use colonoscopy was available. To facilitate early detection of these cases, additional strategies to inform participants about the necessity of follow-up colonoscopy are needed. Interventions to reduce factors related to colonoscopy non-adherence, such as embarrassment, fear of the invasive procedure and examination result should be applied. Other outreach strategies could be built on sending all colonoscopy non-respondents a letter explaining the potential benefits or offering telephone calls to help them schedule colonoscopy appointments.

The success of a CRC screening programme not only depends on test diagnostic performance but to a very large extent also on adherence to screening invitation. The uptake rate was about $7 \%$ in the first year of the Wallonia-Brussels CRC screening programme implementation, whereas much higher participation rate (up to $50 \%$ ) was achieved in the Flemish CRC screening programme. ${ }^{37,41}$ Several differences in screening strategies may explain this very large discrepancy. First, while the target population for screening were individuals aged $50-74$ years in Wallonia and Brussels, people aged 56-74 years were invited for screening in Flanders. According to a prior study, the uptake rate of CRC screening was significantly lower in the age group 50-54 years compared to 55-59 years or older, but the difference was modest (48.0\% vs. 55.6\%). ${ }^{42}$ Second, the population in the Walloon and Brussels-capital region was initially invited merely by a letter without inclusion of a test kit, whereas the Flemish population was invited by an instruction letter along with a FIT kit. Third, in contrast to Flanders, there was no specific reminder for persons who did not collect a test kit from their GPs or who did not return the completed kit in Wallonia and Brussels. These major differences underline once more the overwhelming importance of designing practicalities of screening offers in a way that ensures maximum possible use and maximum possible effects. Even much higher uptake rates $(71 \%)$ have been achieved in the Netherlands, where the success attributes to the implementation of real-time monitoring that allows immediate adjustments to the screening programme. ${ }^{43}$

To our knowledge, our study is the first to comprehensively evaluate the benefits of replacing gFOBT with FIT in a populationbased CRC screening programme by comparing the detection rates, PPVs and incidence rates of gFOBT/FIT interval cancers. Our study population consists of average-risk individuals, comprising the age range commonly invited for CRC screening. Our findings may therefore be relevant for many countries considering implementation or modification of CRC screening programme. In addition, the large sample size enabled estimating key variables of gFOBT and FIT performance at high levels of precision.
Several limitations should be considered when interpreting our results. First, unexpected significant difference in the proportion of follow-up colonoscopy compliance was observed between individuals with positive gFOBT and those with FIT. Prior study also found similar variation and suggested the possible reason could be that gFOBT is a longer-standing screening test, the participants completing this test might be more likely to follow-up with subsequent testing. ${ }^{7}$ Notably, the detection rates of neoplasms were much lower among gFOBT users than FIT users, despite the higher completion rate of follow-up colonoscopy among gFOBT users. This implies that with higher colonoscopy adherence after positive FIT, even larger differences between gFOBT and FIT in detection rates may be observed. Second, as CRC progression may occur after the first months of a positive faecal test, ${ }^{44}$ a 2-year timeframe used to ascertain compliance rate of follow-up colonoscopy may have potentially inflated the number of screen-detected advanced neoplasms; however, given the almost unchanged PPVs for advanced neoplasm and proportion of screen-detected CRCs when considering follow-up colonoscopy as colonoscopy conducted within 6 months after positive faecal test, the impact of this prolonged follow-up window is likely to be limited. Third, colonoscopy information from the insurance claims database did not include important items such as size and number of detected polyps, or the quality of bowel preparation. Fourth, relatively low participation rates might reflect a relevant selfselection of participants, which might limit the generalisability of results to all screening programmes. Finally, we did not exclude FIT users having a history of gFOBT in the analysis on screen- and non-screen-detected CRCs, as we did not directly compare the characteristics of cancers and affected patients between the gFOBT- and FIT-based screening programmes. Also, this most likely reflects real-life conditions when a screening programme is in transition, which may provide better estimates than exclusively focusing on "pure" initial screening by FIT.

Despite these limitations, our study demonstrates that within a completely implemented organised CRC screening programme, replacing gFOBT with FIT strongly improved the advanced neoplasm detection rate and reduced interval cancer incidence. Implementation of FIT-based screening, along with measures to optimise screening adherence, is strongly encouraged in countries where gFOBT-based screening is still employed, or a new programme is in the process of planning.

\section{ACKNOWLEDGEMENTS}

We sincerely thank all the members of the Walloon screening organisation for their efforts on the CRC screening programme implementation. We also thank the Walloon authority Agency for a life of quality for supporting the Walloon CRC screening programme. We gratefully thank all the registration teams of the BCR for the data collection and management. We sincerely thank Ms. Petra Denolf at the BCR for the data preparation for our study.

\section{AUTHOR CONTRIBUTIONS}

Planning the study: F.G., I.D.B. and H.B. Data collection: I.D.B., J.F., M.C., M.P. and L.V.E. Statistical analysis and results interpretation: F.G., I.D.B. and H.B. Drafting the manuscript: F.G. Revision of the manuscript: I.D.B. and H.B. Critical reading and approving the manuscript: all authors.

\section{ADDITIONAL INFORMATION}

Ethics approval and consent to participate Approval for the implementation of the Wallonia-Brussels-organised CRC screening programme and data linkage with the IMA and BCR databases for analyses was provided by the Sectoral Committee of Social Security and Health with reference number 16/019.

Consent to publish All participants within the screening programme completed informed consent, declaring that personal information can be used for scientific research to evaluate and improve the screening programme. All analyses were based 
on the anonymised data and performed within the secured BCR environment, strictly in line with all applicable privacy restrictions. No identifying patient details, images or videos are used in this manuscript.

Data availability Additional summary tables and sensitivity analyses are available upon reasonable request from the corresponding author.

Competing interests The authors declare no competing interests.

Funding information The analyses for this project were supported by grants from the German Cancer Aid (No. 70112095) and the German Federal Ministry of Education and Research (No. 01GL1712). Open Access funding enabled and organized by Projekt DEAL.

Supplementary information is available for this paper at https://doi.org/10.1038/ s41416-020-0754-5.

Publisher's note Springer Nature remains neutral with regard to jurisdictional claims in published maps and institutional affiliations.

\section{REFERENCES}

1. Bray, F., Ferlay, J., Soerjomataram, I., Siegel, R. L., Torre, L. A. \& Jemal, A. Global cancer statistics 2018: GLOBOCAN estimates of incidence and mortality worldwide for 36 cancers in 185 countries. CA Cancer J. Clin. https://doi.org/10.3322/ caac.21492 (2018)

2. Shaukat, A., Mongin, S. J., Geisser, M. S., Lederle, F. A., Bond, J. H., Mandel, J. S. et al. Long-term mortality after screening for colorectal cancer. N. Engl. J. Med. 369, 1106-1114 (2013).

3. Rozen, P., Levi, Z., Hazazi, R., Waked, A., Vilkin, A., Maoz, E. et al. Quantitative colonoscopic evaluation of relative efficiencies of an immunochemical faecal occult blood test and a sensitive guaiac test for detecting significant colorectal neoplasms. Aliment. Pharmacol. Ther. 29, 450-457 (2009).

4. Hol, L., van Leerdam, M. E., van Ballegooijen, M., van Vuuren, A. J., van Dekken, H., Reijerink, J. C. et al. Screening for colorectal cancer: randomised trial comparing guaiac-based and immunochemical faecal occult blood testing and flexible sigmoidoscopy. Gut 59, 62-68 (2010).

5. van Rossum, L. G., van Rijn, A. F., Laheij, R. J., van Oijen, M. G., Fockens, P. \& van Krieken, H. H. et al. Random comparison of guaiac and immunochemical fecal occult blood tests for colorectal cancer in a screening population. Gastroenterology 135, 82-90 (2008).

6. Brenner, H. \& Tao, S. Superior diagnostic performance of faecal immunochemical tests for haemoglobin in a head-to-head comparison with guaiac based faecal occult blood test among 2235 participants of screening colonoscopy. Eur. J. Cancer 49, 3049-3054 (2013).

7. Akram, A., Juang, D., Bustamante, R., Liu, L., Earles, A., Ho, S. B. et al. Replacing the guaiac fecal occult blood test with the fecal immunochemical test increases proportion of individuals screened in a large healthcare setting. Gastroenterol. Hepatol. 15, 1265-1270 and 1261 (2017).

8. Senore, C., Basu, P., Anttila, A., Ponti, A., Tomatis, M., Vale, D. B. et al. Performance of colorectal cancer screening in the European Union Member States: data from the second European screening report. Gut 68, 1232-1244 (2019).

9. Vitellius, C., Laly, M., Banaszuk, A. S., Deherce, I., Cornet, N., Bertrais, S. et al. Contribution of the OC Sensor((R)) immunoassay in comparison to the Hemoccult II((R)) guaiac-test in organized colorectal cancer screening. Eur. J. Epidemiol. 34, 163-172 (2019).

10. Schreuders, E. H., Ruco, A., Rabeneck, L., Schoen, R. E., Sung, J. J. Y., Young, G. P. et al. Colorectal cancer screening: a global overview of existing programmes. Gut 64, 1637-1649 (2015)

11. Cancer Screening in the European Union. Report on the Implementation of the Council Recommendation on Cancer Screening (2017). https://ec.europa.eu/health/ sites/health/files/major_chronic_diseases/docs/2017_cancerscreening_2ndreport implementation_en.pdf. Accessed 17 April 2019.

12. Bretagne, J. F., Piette, C., Cosson, M., Durand, G. \& Lievre, A. Switching from guaiac to immunochemical faecal occult blood test increases participation and diagnostic yield of colorectal cancer screening. Dig. Liver Dis. https://doi.org/10.1016/j. dld.2019.05.004 (2019).

13. Moss, S., Mathews, C., Day, T. J., Smith, S., Seaman, H. E., Snowball, J. et al. Increased uptake and improved outcomes of bowel cancer screening with a faecal immunochemical test: results from a pilot study within the national screening programme in England. Gut 66, 1631-1644 (2017).
14. Steele, R. J., McDonald, P. J., Digby, J., Brownlee, L., Strachan, J. A., Libby, G. et al. Clinical outcomes using a faecal immunochemical test for haemoglobin as a firstline test in a national programme constrained by colonoscopy capacity. U. Eur. Gastroenterol. J. 1, 198-205 (2013).

15. Fraser, C. G., Allison, J. E., Young, G. P., Halloran, S. P. \& Seaman, H. E. Improving the reporting of evaluations of faecal immunochemical tests for haemoglobin: the FITTER standard and checklist. Eur. J. Cancer Prev. 24, 24-26 (2015).

16. Macken, E., Van Dongen, S., Francque, S. \& Van Hal, G. Parameters influencing the quality of colonoscopy in Belgium: a critical evaluation. Acta Gastroenterol. Belg. 81, 29-38 (2018).

17. Cancer Burden in Belgium. https://kankerregister.org/media/docs/publications/ BCR_publicatieCancerBurden2016_web160616.pdf. Accessed 29 May 2019.

18. Samadder, N. J., Curtin, K., Tuohy, T. M., Pappas, L., Boucher, K., Provenzale, D. et al. Characteristics of missed or interval colorectal cancer and patient survival: a population-based study. Gastroenterology 146, 950-960 (2014).

19. Bressler, B., Paszat, L. F., Chen, Z., Rothwell, D. M., Vinden, C. \& Rabeneck, L. Rates of new or missed colorectal cancers after colonoscopy and their risk factors: a population-based analysis. Gastroenterology 132, 96-102 (2007).

20. Sawhney, M. S., Farrar, W. D., Gudiseva, S., Nelson, D. B., Lederle, F. A., Rector, T. S. et al. Microsatellite instability in interval colon cancers. Gastroenterology 131, 1700-1705 (2006).

21. Wilschut, J. A., Habbema, J. D., van Leerdam, M. E., Hol, L., Lansdorp-Vogelaar, I., Kuipers, E. J. et al. Fecal occult blood testing when colonoscopy capacity is limited. J. Natl. Cancer Inst. 103, 1741-1751 (2011).

22. van Rossum, L. G., van Rijn, A. F., Laheij, R. J., van Oijen, M. G., Fockens, P., Jansen, J. B. et al. Cutoff value determines the performance of a semi-quantitative immunochemical faecal occult blood test in a colorectal cancer screening programme. Br. J. Cancer 101, 1274-1281 (2009).

23. Hol, L., Wilschut, J. A., van Ballegooijen, M., van Vuuren, A. J., van der Valk, H., Reijerink, J. C. et al. Screening for colorectal cancer: random comparison of guaiac and immunochemical faecal occult blood testing at different cut-off levels. Br. J. Cancer 100, 1103-1110 (2009).

24. Selby, K., Levine, E. H., Doan, C., Gies, A., Brenner, H., Quesenberry, C. et al. Effect of sex, age and positivity threshold on fecal immunochemical test accuracy: a systematic review and meta-analysis. Gastroenterology https://doi.org/10.1053/j. gastro.2019.08.023 (2019).

25. Vart, G., Banzi, R. \& Minozzi, S. Comparing participation rates between immunochemical and guaiac faecal occult blood tests: a systematic review and metaanalysis. Prev. Med. 55, 87-92 (2012).

26. Duffy, M. J., van Rossum, L. G., van Turenhout, S. T., Malminiemi, O., Sturgeon, C., Lamerz, R. et al. Use of faecal markers in screening for colorectal neoplasia: a European group on tumor markers position paper. Int. J. Cancer 128, 3-11 (2011).

27. Garcia, M., Domenech, X., Vidal, C., Torne, E., Mila, N. \& Binefa, G. et al. Interval cancers in a population-based screening program for colorectal cancer in cataIonia, Spain. Gastroenterol. Res. Pract. https://doi.org/10.1155/2015/672410 (2015).

28. Kronborg, O., Fenger, C., Olsen, J., Jorgensen, O. D. \& Sondergaard, O. Randomised study of screening for colorectal cancer with faecal-occult-blood test. Lancet 348, 1467-1471 (1996).

29. Faivre, J., Dancourt, V., Lejeune, C., Tazi, M. A., Lamour, J., Gerard, D. et al. Reduction in colorectal cancer mortality by fecal occult blood screening in a French controlled study. Gastroenterology 126, 1674-1680 (2004).

30. Blom, J. \& Tornberg, S. Interval cancers in a guaiac-based colorectal cancer screening programme: consequences on sensitivity. J. Med. Screen. 24, 146-152 (2017).

31. Steele, R. J., McClements, P., Watling, C., Libby, G., Weller, D., Brewster, D. H. et al. Interval cancers in a FOBT-based colorectal cancer population screening programme: implications for stage, gender and tumour site. Gut 61, 576-581 (2012).

32. van der Vlugt, M., Grobbee, E. J., Bossuyt, P. M. M., Bos, A., Bongers, E., Spijker, W. et al. Interval colorectal cancer incidence among subjects undergoing multiple rounds of fecal immunochemical testing. Gastroenterology 153, 439-447 and 432 (2017).

33. Mlakar, D. N., Bric, T. K., Skrjanec, A. L. \& Krajc, M. Interval cancers after negative immunochemical test compared to screen and non-responders' detected cancers in Slovenian colorectal cancer screening programme. Radiol. Oncol. 52, 413-421 (2018).

34. Parente, F., Boemo, C., Ardizzoia, A., Costa, M., Carzaniga, P., llardo, A. et al. Outcomes and cost evaluation of the first two rounds of a colorectal cancer screening program based on immunochemical fecal occult blood test in northern Italy. Endoscopy 45, 27-34 (2013).

35. Digby, J., Fraser, C. G., Carey, F. A., Lang, J., Stanners, G. \& Steele, R. J. Interval cancers using a quantitative faecal immunochemical test (FIT) for haemoglobin when colonoscopy capacity is limited. J. Med. Screen. 23, 130-134 (2016).

36. Wieten, E., Schreuders, E. H., Grobbee, E. J., Nieboer, D., Bramer, W. M., LansdorpVogelaar, I. et al. Incidence of faecal occult blood test interval cancers in 
population-based colorectal cancer screening: a systematic review and metaanalysis. Gut https://doi.org/10.1136/gutjnl-2017-315340 (2018).

37. Monitoring report of the Flemish Colorectal Cancer Screening Programme (2017). https://dikkedarmkanker.bevolkingsonderzoek.be/sites/default/files/atoms/files/ Jaarrapport2017_DEF_0.pdf. Accessed 19 April 2019.

38. Brenner, H., Chang-Claude, J., Seiler, C. M. \& Hoffmeister, M. Interval cancers after negative colonoscopy: population-based case-control study. Gut 61, 1576-1582 (2012).

39. Nally, D. M., Ballester, A. W., Valentelyte, G. \& Kavanagh, D. O. The contribution of endoscopy quality measures to the development of interval colorectal cancers in the screening population: a systematic review. Int. J. Colorec. Dis. 34, 123-140 (2019).

40. Butterly, L. F., Nadel, M. R., Anderson, J. C., Robinson, C. M., Weiss, J. E., Lieberman D. et al. Impact of colonoscopy bowel preparation quality on follow-up interval recommendations for average-risk patients with normal screening colonoscopies: data from the new hampshire colonoscopy registry. J. Clin. Gastroenterol. https:// doi.org/10.1097/MCG.0000000000001115 (2018).

41. Klabunde, C., Blom, J., Bulliard, J. L., Garcia, M., Hagoel, L., Mai, V. et al. Participation rates for organized colorectal cancer screening programmes: an international comparison. J. Med. Screen. 22, 119-126 (2015).

42. Quyn, A. J., Fraser, C. G., Stanners, G., Carey, F. A., Carden, C., Shaukat, A. et al. Uptake trends in the Scottish Bowel Screening Programme and the influences of age, sex, and deprivation. J. Med. Screen. 25, 24-31 (2018).
43. Toes-Zoutendijk, E., van Leerdam, M. E., Dekker, E., van Hees, F., Penning, C., Nagtegaal, I. et al. Real-time monitoring of results during first year of dutch colorectal cancer screening program and optimization by altering fecal immunochemical test cut-off levels. Gastroenterology 152, 767-775 and 762 (2017).

44. Corley, D. A., Jensen, C. D., Quinn, V. P., Doubeni, C. A., Zauber, A. G., Lee, J. K. et al. Association between time to colonoscopy after a positive fecal test result and risk of colorectal cancer and cancer stage at diagnosis. JAMA 317, 1631-1641 (2017). Attribution 4.0 International License, which permits use, sharing, adaptation, distribution and reproduction in any medium or format, as long as you give appropriate credit to the original author(s) and the source, provide a link to the Creative Commons license, and indicate if changes were made. The images or other third party material in this article are included in the article's Creative Commons license, unless indicated otherwise in a credit line to the material. If material is not included in the article's Creative Commons license and your intended use is not permitted by statutory regulation or exceeds the permitted use, you will need to obtain permission directly from the copyright holder. To view a copy of this license, visit http://creativecommons. org/licenses/by/4.0/.

(c) The Author(s) 2020, corrected publication 2021 